\title{
Cardiopulmonary rehabilitation for obese sleep-disordered breathing: a new treatment frontier?
}

\author{
Craig L Phillips, ${ }^{1,2,3}$ Elizabeth A Cayanan, ${ }^{1,4}$ Camilla M Hoyos ${ }^{1,5}$
}

The health benefits of routine exercise in otherwise healthy people, including in those who are obese, are well proven. ${ }^{1}$ Exercise improves cardiorespiratory fitness and improves blood supply to the musculature to enhance oxidative metabolism and overall efficiency of energy expenditure. There is also evidence showing the benefits of exercise might extend to people with debilitating conditions including congestive heart failure $(\mathrm{CHF}),{ }^{2} \mathrm{COPD}^{3}$ and more recently, obesity hypoventilation syndrome (OHS). ${ }^{4}$ In these populations, exercise training as part of a rehabilitation programme has been shown to improve exercise and functional capacity as well as overall quality of life. However, there are inherent (disease-specific) physiological limitations that pose significant barriers to full engagement in exercise training in people with chronic illness. For example, in the setting of pulmonary rehabilitation in COPD, exercise-induced dynamic hyperinflation increases the work of breathing, resulting in severe dyspnoea and a reduction in exercise tolerance, making it difficult for patients to adhere to exercise programmes. ${ }^{5}$ As a solution to this, rehabilitation programmes, particularly in COPD, are increasingly using supportive methods in an attempt to reduce the physiological burden of exercise and improve exercise tolerance and capacity with anticipated positive effects on health outcomes.

For example, non-invasive ventilation (NIV) that is deployed during exercise in patients with COPD undergoing pulmonary rehabilitation is thought to unload respiratory muscles and reduce the work

\footnotetext{
${ }^{1}$ Centre for Sleep and Chronobiology, Woolcock Institute of Medical Research, University of Sydney, Glebe, New South Wales, Australia

${ }^{2}$ Department of Respiratory and Sleep Medicine, Royal North Shore Hospital, St Leonards, New South Wales, Australia

${ }^{3}$ Sydney Medical School, University of Sydney, Sydney, New South Wales, Australia

${ }^{4}$ Sydney Nursing School, University of Sydney, Sydney, New South Wales, Australia

${ }^{5}$ School of Psychology, Faculty of Science, University of Sydney, Sydney, New South Wales, Australia
}

Correspondence to Dr Camilla M Hoyos, School of Psychology, University of Sydney, Sydney, New South Wales 2050, Australia; camilla.hoyos@sydney.edu.au of breathing, reducing diaphragm fatigue and dyspnoea. While this is supported by a number of studies showing an increase in training intensity, the effect on subsequent exercise capacity seems variable and currently the benefits remain unclear. ${ }^{6}$ Another technique used to improve exercise tolerance involves respiratory muscle training (RMT), incorporating both respiratory muscle strength (resistive/threshold) and respiratory endurance (isocapnic hyperpnoea) training. In healthy individuals, RMT improves exercise capacity ${ }^{78}$ and, when deployed in $\mathrm{COPD}^{9}$ and $\mathrm{CHF}^{10}$ rehabilitation programmes, increases respiratory muscle strength to enhance exercise capacity.

Obesity is another condition where exercise tolerance and capacity can be limited, and in a small number of obesity intervention studies, the use of RMT has resulted in improved exercise capacity. ${ }^{11-13}$ NIV, on the other hand, has not been extensively explored as an adjunct to exercise in the obese, although one small study did find that proportional assist ventilation (a form of NIV) delivered during exercise, increased exercise endurance by more than $30 \%$ in over half the patients. ${ }^{14}$ Given these positive, although limited data supporting the concept of RMT and NIV to improve exercise capacity in obesity, it seems appropriate that both are explored as part of rehabilitation programmes in obesity-related sleep-disordered breathing (SDB) including OHS and obstructive sleep apnoea (OSA) where exercise capacity is limited. ${ }^{15}$

Interestingly, a recent study in OHS found that NIV (but not CPAP) during sleep resulted in a small but clinically insignificant increase in exercise capacity measured with the 6 minute walk test (6MWT). ${ }^{16}$ Furthermore, a recent pilot randomised controlled trial (RCT) in OHS found clinically important shortterm improvements in exercise capacity (6MWT) when a rehabilitation programme involving diet and exercise was added to nocturnal NIV. ${ }^{4}$ However, the use of NIV during exercise or RMT as part of a rehabilitation programme has not been extensively explored in SDB. One small RCT in patients with untreated OSA recently assessed the impact of RMT involving 12 weeks of moderate intensity inspiratory muscle training on exercise capacity but found no effect. ${ }^{17}$ Nevertheless, given the potential for RMT and/or NIV to improve exercise capacity and health outcomes, these aids should be more rigorously explored in obese patients with SDB.

It is therefore timely that a recent RCT published in Thorax explored this very concept. ${ }^{18}$ Vivodtzev et al studied 53 morbidly obese (body mass index $38 \mathrm{~kg}$ / $\mathrm{m}^{2}$ ) patients with severe OSA (Apnea Hypopnea Index $(\mathrm{AHI})>30 / \mathrm{h}$ ) who were established on CPAP and who were highly compliant (>6.5 hours/night). ${ }^{18}$ Patients with unstable cardiovascular disease or with orthopaedic or neurological disease that would limit participation in the rehabilitation programme were excluded. Eligible patients were then randomised to cycling exercise training alone (ERGO), or ERGO combined with either NIV $(\mathrm{ERGO}+\mathrm{NIV})$ or RMT (ERGO+RMT). All patients underwent three exercise training sessions per week (up to $45 \mathrm{~min}$ of cycling) over a 3-month period (36 sessions total). The ERGO+RMT patients performed up to $2 \times 15 \mathrm{~min}$ RMT sessions after ERGO exercise. RMT involved isocapnic hyperventilation where patients were kept at 50\% maximum voluntary ventilation. This required breathing rates of up to 48 breaths/min at tidal volumes of $\sim 50 \%$ of FVC. The ERGO-NIV group received bi-level positive airway pressure during ERGO sessions via a full-face mask set at mean inspiratory/expiratory pressures (IPAP/EPAP) of 19/4 $\mathrm{cmH}_{2} \mathrm{O}$. The primary outcome was the $6 \mathrm{~min}$ walking distance based on a standard 6MWT. ${ }^{19}$ Aerobic capacity measured via a Cardio-Pulmonary Exercise Test (CPET), metabolic and inflammatory markers, quality of life, physical activity and sleep quality outcomes were also assessed.

Overall, the study found a small increase in 6MWT distance of around $25 \mathrm{~m}$ in all groups. The American Thoracic Society criteria suggest that in COPD, a minimum improvement of $>70 \mathrm{~m}$ is likely to be clinically important. ${ }^{19}$ Consequently, the small improvement found in this study, in patients presumably without lung disease, is not likely to be clinically important. However, the CPET results demonstrated higher increases in peak $\mathrm{VO}_{2}(\sim 2-3 \mathrm{~mL} / \mathrm{min} / \mathrm{kg})$ in the $\mathrm{ERGO}+\mathrm{NIV}$ and ERGO+RMT groups, reflecting a greater aerobic capacity. Assuming a mean body weight of around $120 \mathrm{~kg}$, this increase appears to amount to approximately $10 \%$ of baseline $\mathrm{VO}_{2}$ which, in contrast to the $6 \mathrm{MWT}$, is likely 
to be clinically important. Of interest, the ERGO+RMT group also had higher levels of VE during CPET. While the authors argue that this likely reflects greater respiratory muscle capacity, it also suggests it comes at a cost with greater ventilatory inefficiency to achieve a similar peak $\mathrm{VO}_{2}$ to the ERGO+NIV group. Although the exact mechanisms are not apparent, possibilities include worse ventilation/ perfusion matching and increased ventilatory response to exercise. Among the secondary outcomes, there were improvements in arterial stiffness (pulse wave velocity), lipids (high-density lipoprotein and low-density lipoprotein) and in markers of insulin resistance in all three groups (with no intergroup differences) suggesting an overall benefit from ERGO independent of NIV or RMT, as would be expected with consistent cardiorespiratory exercise. Self-measured blood pressure was markedly reduced by $\sim 9 / 6 \mathrm{~mm}$ $\mathrm{Hg}$ in the ERGO+NIV group, however it is unclear why this improvement did not occur across all groups.

One potential limitation with this study was the exclusion of patients who were not highly compliant with CPAP use. This makes the findings non-generalisable to the wider OSA population where compliance in the real world may be far more variable. It therefore does not tell us whether the methods used in this study could also be deployed in less compliant patients and whether they would have a positive effect. It also does not tell us the extent to which physiological changes associated with near-perfect control of OSA might have influenced exercise tolerance and capacity in this study. For example, nocturnal NIV in $\mathrm{COPD}^{20}$ and $\mathrm{OHS}^{16}$ has been associated with exercise improvements.

Despite the study not establishing a positive effect of NIV or RMT (on top of ERGO exercise) to increase exercise capacity as measured by the 6MWT, it does nevertheless leave the door open for further exploration of adjuncts for exercise as part of cardiopulmonary rehabilitation programmes in obese patients with SDB. As the authors discuss, this may be particularly relevant in the setting of OHS where both nocturnal NIV $^{16}$ and exercise ${ }^{4}$ may have positive effects on exercise capacity. Ultimately, a multipronged approach involving weight loss, NIV (or CPAP) delivered during sleep, NIV delivered during exercise and RMT delivered in parallel may prove to be highly effective in improving health outcomes in obese SDB populations.
However, there may be differences in the effectiveness of both NIV and $\mathrm{RMT}^{7}$ based on the mode and type of delivery, and further mechanistic studies are needed to tailor therapies for different patients based on their phenotypic characteristics. In addition, growing evidence suggests that SDB treatment may be a crucial component of a successful weight loss programme ${ }^{21}$ where it could have a synergistic effect on weight loss and associated improvements in cardiometabolic health outcomes. ${ }^{22}$ This is clearly a research agenda item that the current authors are well placed to explore in future RCTs and as such, they should be commended for starting a very important line of enquiry that could change the landscape for SDB treatment in the future.

Funding The authors have not declared a specific grant for this research from any funding agency in the public, commercial or not-for-profit sectors.

\section{Competing interests None declared.}

Patient consent Not required.

Provenance and peer review Commissioned; internally peer reviewed.

(c) Article author(s) (or their employer(s) unless otherwise stated in the text of the article) 2018. All rights reserved. No commercial use is permitted unless otherwise expressly granted.

\section{Check for updates}

To cite Phillips CL, Cayanan EA, Hoyos CM. Thorax 2018;73:603-604.

Accepted 5 March 2018

Published Online First 23 March 2018

\section{(5) Linked}

http://dx.doi.org/10.1136/thoraxjnl-2017-211152

Thorax 2018;73:603-604.

doi:10.1136/thoraxjnl-2018-211607

\section{REFERENCES}

1 Lavie CJ, De Schutter A, Milani RV. Healthy obese versus unhealthy lean: the obesity paradox. Nat Rev Endocrinol 2015;11:55-62.

2 Adams V, Niebauer J. Reversing heart failureassociated pathophysiology with exercise: what actually improves and by how much? Heart Fail Clin 2015;11:17-28

3 Paneroni M, Simonelli C, Vitacca M, et al. Aerobic exercise training in very severe chronic obstructive pulmonary disease: a systematic review and metaanalysis. Am J Phys Med Rehabil 2017:96:541-8.

4 Mandal S, Suh ES, Harding R, et al. Nutrition and Exercise Rehabilitation in Obesity hypoventilation syndrome (NERO): a pilot randomised controlled trial. Thorax 2018;73:62-9.

5 Ambrosino N, Cigni P. Non invasive ventilation as an additional tool for exercise training. Multidiscip Respir Med 2015;10:14.
6 Menadue C, Piper AJ, van 't Hul AJ, et al. Non-invasive ventilation during exercise training for people with chronic obstructive pulmonary disease. Cochrane Database Syst Rev 2014;5:CD007714.

7 Illi SK, Held U, Frank I, et al. Effect of respiratory muscle training on exercise performance in healthy individuals: a systematic review and meta-analysis. Sports Med 2012:42:707-24.

8 Sales AT, Fregonezi GA, Ramsook AH, et al. Respiratory muscle endurance after training in athletes and nonathletes: a systematic review and meta-analysis. Phys Ther Sport 2016;17:76-86.

9 Bernardi E, Pomidori L, Bassal F, et al. Respiratory muscle training with normocapnic hyperpnea improves ventilatory pattern and thoracoabdominal coordination, and reduces oxygen desaturation during endurance exercise testing in COPD patients. Int J Chron Obstruct Pulmon Dis

2015; 10:1899-906.

10 Cahalin LP, Arena R, Guazzi M, et al. Inspiratory muscle training in heart disease and heart failure: $a$ review of the literature with a focus on method of training and outcomes. Expert Rev Cardiovasc Ther 2013;11:161-77.

11 Salvadego D, Sartorio A, Agosti F, et al. Respiratory muscle endurance training reduces the 02 cost of cycling and perceived exertion in obese adolescents. Am J Physiol Regul Integr Comp Physiol 2017:313:R487-95.

12 Villiot-Danger JC, Villiot-Danger E, Borel JC, et al. Respiratory muscle endurance training in obese patients. Int J Obes 2011;35:692-9.

13 Frank I, Briggs R, Spengler CM. Respiratory muscles, exercise performance, and health in overweight and obese subjects. Med Sci Sports Exerc 2011;43:714-27.

14 Dreher M, Kabitz HJ, Burgardt V, et al. Proportional assist ventilation improves exercise capacity in patients with obesity. Respiration 2010;80:106-11.

15 Beitler JR, Awad KM, Bakker JP, et al. Obstructive sleep apnea is associated with impaired exercise capacity: a cross-sectional study. J Clin Sleep Med 2014;10:1199-204.

16 Masa JF, Corral J, Alonso ML, et al. Efficacy of different treatment alternatives for obesity hypoventilation syndrome. Pickwick study. Am J Respir Crit Care Med 2015;192:86-95.

17 Souza AKF, Dornelas de Andrade A, de Medeiros AIC, et al. Effectiveness of inspiratory muscle training on sleep and functional capacity to exercise in obstructive sleep apnea: a randomized controlled trial. Sleep Breath 2017.

18 Vivodtzev I, Tamisier R, Croteau M, et al. Ventilatory support or respiratory muscle training as adjuncts to exercise in obese CPAP-treated patients with obstructive sleep apnoea: a randomised controlled trial. Thorax 2018;73:634-43.

19 ATS Committee on Proficiency Standards for Clinical Pulmonary Function Laboratories. ATS statement: guidelines for the six-minute walk test. Am J Respir Crit Care Med 2002;166:111-7.

20 Kneidinger N, Gloeckl R, Schönheit-Kenn U, et al. Impact of nocturnal noninvasive ventilation on pulmonary rehabilitation in patients with end-stage lung disease awaiting lung transplantation. Respiration 2017.

21 Borel AL, Leblanc X, Alméras N, et al. Sleep apnoea attenuates the effects of a lifestyle intervention programme in men with visceral obesity. Thorax 2012:67:735-41

22 Chirinos JA, Gurubhagavatula I, Teff K, et al. CPAP, weight loss, or both for obstructive sleep apnea. $N$ Engl J Med 2014;370:2265-75. 